\title{
Multispectral imaging of clinically relevant cellular targets in tonsil and lymphoid tissue using semiconductor quantum dots
}

\author{
Thomas J Fountaine ${ }^{1, *}$, Stephen M Wincovitch ${ }^{2, *}$, David H Geho ${ }^{3}$, Susan H Garfield ${ }^{2}$ \\ and Stefania Pittaluga ${ }^{1}$
}

${ }^{1}$ Laboratory of Pathology, National Cancer Institute, National Institutes of Health, Bethesda, MD, USA; ${ }^{2} C C R$ Confocal Microscopy Core Facility, Laboratory of Experimental Carcinogenesis, National Cancer Institute, National Institutes of Health, Bethesda, MD, USA and ${ }^{3}$ Center for Applied Proteonomics and Molecular Medicine, George Mason University, Manassas, VA, USA

\begin{abstract}
Determination of the expression and spatial distribution of molecular epitopes, or antigens, in patient tissue specimens has substantially improved the pathologist's ability to classify disease processes. Certain disease pathophysiologies are marked by characteristic increased or decreased expression of developmentally controlled antigens, defined as Cluster of Differentiation markers, that currently form the foundation for understanding lymphoid malignancies. While chromogens and organic fluorophores have been utilitized for some time in immunohistochemical analyses, developments in synthetic, inorganic fluorophore semiconductors, namely quantum dots, offer a versatile alternative reporter system. Quantum dots are stable fluorophores, are resistant to photobleaching, and are attributed with wide excitation ranges and narrow emission spectra. To date, routinely processed, formalin-fixed tissues have only been probed with two quantum dot reporters simultaneously. In the present study, streptavidin-conjugated quantum dots with distinct emission spectra were tested for their utility in identifying a variety of differentially expressed antigens (surface, cytoplasmic, and nuclear). Slides were analyzed using confocal laser scanning microscopy, which enabled with a single excitation wavelength $(488 \mathrm{~nm}$ argon laser) the detection of up to seven signals (streptavidin-conjugated quantum dots 525, 565, 585, 605, 655, 705 and $805 \mathrm{~nm}$ ) plus the detection of 4'6-DiAmidino-2-Phenyllndole with an infra-red laser tuned to $760 \mathrm{~nm}$ for two photon excitation. Each of these signals was specific for the intended morphologic immunohistochemical target. In addition, five of the seven streptavidin-conjugated quantum dots tested (not streptavidin-conjugated quantum dots 585 or $805 \mathrm{~nm}$ ) were used on the same tissue section and could be analyzed simultaneously on routinely processed formalin-fixed, paraffin-embedded sections. Application of this multiplexing method will enable investigators to explore the clinically relevant multidimensional cellular interactions that underlie diseases, simultaneously.

Modern Pathology (2006) 19, 1181-1191. doi:10.1038/modpathol.3800628; published online 16 June 2006
\end{abstract}

Keywords: immunofluorescence; quantum dots; immunohistochemistry; multiplexing; multispectral; confocal microscopy

For some time, the application of antibodies in the immunohistochemical staining of tissues has markedly improved the classification and diagnosis of disease processes. Nowhere is this more evident than in the lymphoid malignancies, wherein the identification of differentiation antigens, also called

Correspondence: Dr S Pittaluga, MD, PhD, Laboratory of Pathology, National Cancer Institute, Bldg 10, Room 2N109, 10 Center Drive, Bethesda, MD 20892, USA.

E-mail: stefpitt@mail.nih.gov

${ }^{*}$ Both authors have contributed equally to this project.

Received 1 February 2006; revised and accepted 12 April 2006; published online 16 June 2006
CD markers, has fundamentally improved classification of diseases and elucidated the underlying pathophysiology. A constant infusion of new technologies into pathology practice has enabled these changes, including monoclonal antibodies, streptavidin/biotin interactions, antigen retrieval, organic fluorophores, and enzymatic amplification strategies. A novel nanotechnology, the quantum dot, an inorganic fluorophore, promises to offer the next technological breakthrough in the imaging of patient tissues. Quantum dots have recently been cited as having substantial advantages over traditional organic fluorophores for use in a variety of biological applications including both in vivo imaging and 
in vitro assays. ${ }^{1-3}$ Beneficial properties of quantum dots over organic fluorophores are narrow emission band peaks, broad absorption spectra, intense signals, and remarkable resistance to photobleaching, ${ }^{1,4-8}$ which make them highly useful for the immunohistochemical staining of fixed tissue sections. Not surprisingly, multiple groups have reported the utilization of quantum dots in a variety of biological assays/applications. ${ }^{9-15}$ A significant recent technological advance in quantum dot technologies is pegylation or the introduction of poly (ethylene glycol) groups onto the streptavidinquantum dot conjugates, which substantially decreased nonspecific protein:quantum dot interactions. ${ }^{16,17}$ However, to date, quantum dots reporter technology used in fixed tissue sections has been limited. ${ }^{15,18}$ Several issues have been raised about pitfalls and limited applicability of this technology to routinely processed formalin-fixed, paraffin-embedded tissue sections, ${ }^{7}$ such as lack of target availability and lack of multiple target binding resulting from steric hindrances due to quantum dot size.

Furthermore, the simultaneous detection of three or more multiple targets has yet to be reported. By interfacing quantum dot technology with immunohistochemical staining methodologies and versatile imaging systems, investigators have the ability to identify multiple targets of interest which will allow for: (1) elucidation of complex cell-to-cell and protein-to-protein interactions, (2) clarification of spatial arrangements of cells within a tissue, (3) localization of multiple proteins within a cell, and finally (4) identification of complex protein expression patterns.

In this study, quantum dots ${ }^{\circledR}$ technology has been applied successfully to detect multiple targets on a fixed tissue section. Specifically, the visualization of morphological characteristics, which define the immunological microanatomy of human lymphoid tissue, was investigated through multispectral staining of common lymphoid markers. By combining quantum dots technology and a multispectral imaging system, many of the obstacles of traditional fluorophore staining were overcome and the ability to simultaneously detect multiple targets on formalin-fixed tissue sections was demonstrated.

\section{Materials and methods}

\section{Immunohistochemistry}

Staining was performed on formalin-fixed, paraffinembedded tissue tonsil sections approximately 3$4 \mu \mathrm{m}$ in thickness. For single stains, antigen retrieval methods were performed according to basic immunohistochemistry protocols specific for each antigen. Table 1 summarizes antibodies used, source, dilution, and retrieval conditions. Streptavidinconjugated quantum dots $(525,565,585,605,655$, 705 , and $805 \mathrm{~nm})(2 \mu \mathrm{M}$ stock solution) were purchased from Quantum Dot Corp., Hayward, CA, USA and were used at a final concentration of $6 \mathrm{nM}$ in PBS with $2 \%$ albumin. Generally, after deparaffinization, slides were placed in a microwavable pressure cooker (Nordic Ware, Minneapolis, MN, USA) containing $1 \mathrm{l}$ of $1 \times$ 'Target Retrieval Solution' (Dako, Carpinteria, CA, USA), and heated in a $1100 \mathrm{~W}$ microwave for $40 \mathrm{~min}$ or for $8 \mathrm{~min}$ (hot start). Alternatively to using a microwavable pressure cooker, slides were placed in a steamer for $30 \mathrm{~min}$ using 'Target Retrieval Solution, High pH' (Dako, Carpinetria, CA, USA). After retrieval, slides were incubated in Tris-buffered saline ( $\mathrm{pH}$ 7.6) containing $3 \%$ goat serum for $15 \mathrm{~min}$. Primary antibodies were incubated for $30 \mathrm{~min}$ or overnight at room temperature, then rinsed in Tris-buffered saline ( $\mathrm{pH}$ 7.6) for $15 \mathrm{~min}$ (three times for $5 \mathrm{~min}$ each $(3 \times 5 \mathrm{~min})$ ). A cocktail of biotinylated secondary anti-mouse/antirabbit/anti-goat antibody (Dako, Carpinteria, CA, USA) was applied and incubated for $30 \mathrm{~min}$ at room temperature. Slides were washed in Tris-buffered saline ( $\mathrm{pH} 7.6)$ for $15 \mathrm{~min}(3 \times 5 \mathrm{~min})$ and then incubated with individual streptavidin-conjugated

Table 1 List of antibodies used with quantum dots and retrieval conditions

\begin{tabular}{|c|c|c|c|c|}
\hline Antibody & Clone & Manufacturer & Pretreatment & Dilution \\
\hline $\mathrm{CD} 20$ & L-26 & Dako & $\mathrm{PC} / \mathrm{MW}$ & $1: 200$ \\
\hline CD3 & SP7 & NeoMarkers, LabVision & S/high pH & $1: 500$ \\
\hline BCL-6 & PG-B6p & Dako & $\mathrm{PC} / \mathrm{MW}$ & $1: 20$ \\
\hline CD62/P-selectin & C34 & Vision Biosystems, Novacastra & $\mathrm{PC} / \mathrm{MW}$ & $1: 100$ \\
\hline CD68 & $\mathrm{KP}-1$ & Dako & $\mathrm{PC} / \mathrm{MW}$ & $1: 200$ \\
\hline Cytokeratin, high molecular weight & $34 \beta \mathrm{E} 12$ & Dako & $\mathrm{PC} / \mathrm{MW}$ & $1: 100$ \\
\hline IgD & Polyclonal & Dako & S/high pH & 1:1000 \\
\hline MUM1 & MUM-1p & Dako & $\mathrm{PC} / \mathrm{MW}$ & $1: 200$ \\
\hline Ki-67 & Mib-1 & Dako & $\mathrm{PC} / \mathrm{MW}$ & $1: 100$ \\
\hline Pax-5 & 24 & BD Biosciences, Pharmingen & S/high pH & $1: 100$ \\
\hline S-100 & 15E2E2 & BioGeneX & PC/MW & 1:4000 \\
\hline
\end{tabular}

$\mathrm{PC} / \mathrm{MW}=$ pressure cooker/microwave; $\mathrm{S}=$ steamer.

Dako, Carpinteria, CA, USA; NeoMarkers, LabVision, Fremont, CA, USA; Vision Biosystems Novocastra, Norwell, MA, USA; BD Biosciences, Pharmingen, San Jose, CA, USA; BioGeneX, San Ramon, CA, USA. 
quantum dots for $30 \mathrm{~min}$ at room temperature. After rinsing in Tris-buffered saline ( $\mathrm{pH} \mathrm{7.6)} \mathrm{for} 15 \mathrm{~min}$ $(3 \times 5 \mathrm{~min})$, the slides were air dried and mounted in aqueous mounting media with 4'6-DiAmidino-2PhenylIndole (DAPI) (Vector Laboratories, Inc., Burlingame, CA, USA). Slides were left overnight at room temperature in the dark.

For the sequential multiple staining, an extra avidin-biotin block was included. Avidin block was applied for $10 \mathrm{~min}$, followed by a rinse in Trisbuffered saline ( $\mathrm{pH}$ 7.6) and a 10 min incubation with biotin block (Dako, Carpinteria, CA, USA). These extra blocking steps were performed between the end of the first full detection (ie primary antibody, secondary antibody, quantum dot) and the beginning of the next sequential primary.

\section{Confocal Microscopy}

Images were acquired using a Zeiss LSM 510 NLO confocal system mounted on a Zeiss Axiovert 200M microscope with a Plan Apochromat $20 \times / 0.75$ NA, an oil immersion Plan-Neofluar $40 \times / 1.3$ NA DIC, or an oil immersion Plan-Apochromat $63 \times / 1.4$ NA DIC objective lens. Excitation of streptavidinconjugated quantum dots was performed using the $488 \mathrm{~nm}$ line from a $30 \mathrm{~mW}$ Argon laser while excitation of DAPI was performed by tuning the two photon laser to $760 \mathrm{~nm}$. The DAPI emission was collected with a photo multiplier tube (PMT) using a Band Pass 390-465 nm Infra-Red blocked filter. The assorted streptavidin-conjugated quantum dot emissions were collected with the Zeiss Meta detector. All streptavidin-conjugated quantum dots were collected using custom Meta band pass filters as follows: $505-526 \mathrm{~nm}$ for streptavidin-conjugated quantum dot $525,558-580 \mathrm{~nm}$ for streptavidinconjugated quantum dot 565, 590-612 nm for streptavidin-conjugated quantum dot 605, 633-677 nm for streptavidin-conjugated quantum dot 655, and $687-719 \mathrm{~nm}$ for streptavidin-conjugated quantum dot 705. Multi-track/frame switching configurations were used to minimize any bleed through effect from multiplexing closely emitting streptavidinconjugated quantum dots. For the quintuplet staining, pinholes ranged from 117 to $121 \mu \mathrm{m}$ with optical slices of $1.5 \mu \mathrm{m}$, except for DAPI where the pinhole was maximum or $1000 \mu \mathrm{m}$. Initial acquisitions were collected sequentially for all four tracks (six channels) using the Zeiss AIM software version 3.2 sp2 (Carl Zeiss GmbH, Heidelberg, Germany). All confocal images were acquired with a frame size of 512 by 512 pixels, with a scan zoom ranging from 0.7 to 2 and were averaged four times. Lambda stacks were collected at various emission wavelengths using the two-photon laser tuned to $760 \mathrm{~nm}$ or the $488 \mathrm{~nm}$ line of the Argon laser. Emissions from the streptavidin-conjugated quantum dots plus DAPI were collected using the Zeiss Meta detector (spectral separator) with one filter $(395-715 \mathrm{~nm}$ ) and
$10.7 \mathrm{~nm}$ step size. Lambda scans were acquired at each phase of the project for single, double, triple, and quad-labeled tissue sections.

\section{Results}

In order to test the applicability of a highly multiplexed quantum dot reporter system to routinely processed formalin-fixed, paraffin-embedded tissue, tissue sections from human reactive tonsils and a series of well-known and well-characterized antibodies that recognize specific cellular targets within lymphoid tissue were used. This system was chosen because lymphoid tissue offers a significant interpretive challenge due to the high cellular density and close spatial relationship of different morphological elements. In particular, we focused on the germinal center region, which is the hallmark of a T-cell-dependent antibody response ${ }^{19}$ (Figure 1). The germinal center is the site of B-cell clonal expansion, somatic hypermutation, isotype switching, affinity maturation, apoptosis, plasma cell and memory cell commitment. For these purposes, a vast array of well-characterized antibodies, which are routinely used in clinical practice, is available. The initial goal was to define the optimal conditions for the pegylated streptavidin-conjugated quantum dots to label specific cell surface and nuclear targets, and for this purpose CD20 and MIB-1 (Ki-67 equivalent in formalin-fixed, paraffin-embedded tissue $^{20}$ ) antigens were selected. CD20 is a pan B-cell surface antigen, routinely used in clinical diagnosis, which identifies the majority of B cells in a tonsil with a characteristic distribution, that is, reactive follicles composed of germinal centers and mantles (Figure 1) as well as interfollicular B cells. MIB-1 is a nuclear antigen associated with proliferation, but is not cell specific. The staining pattern of MIB-1 in human tonsil is also well known and highlights the proliferative area of germinal centers, known as the dark zone, as well as any other proliferating cells in the interfollicular areas and the basal layer of the surface epithelium. ${ }^{21}$ For each antibody, the same retrieval conditions were used that were previously determined in our clinical laboratory as optimal for routine chromogenic detection (Table 1). After the application of the appropriate biotinylated secondary antibodies (goat anti-mouse in these two instances), individual streptavidin-conjugated quantum dots of different wavelengths (quantum dot $525 \mathrm{~nm}$, quantum dot $565 \mathrm{~nm}$, quantum dot $585 \mathrm{~nm}$, quantum dot $605 \mathrm{~nm}$, quantum dot $655 \mathrm{~nm}$, quantum dot $705 \mathrm{~nm}$, and quantum dot $805 \mathrm{~nm}$ ) were used. The expected staining pattern was observed for each condition (data from singles not shown). The stability of the conjugates was tested by reanalyzing previously stained tissue sections using the same parameters (ie confocal configuration) as in the original experiment, identical and different lot numbers of 


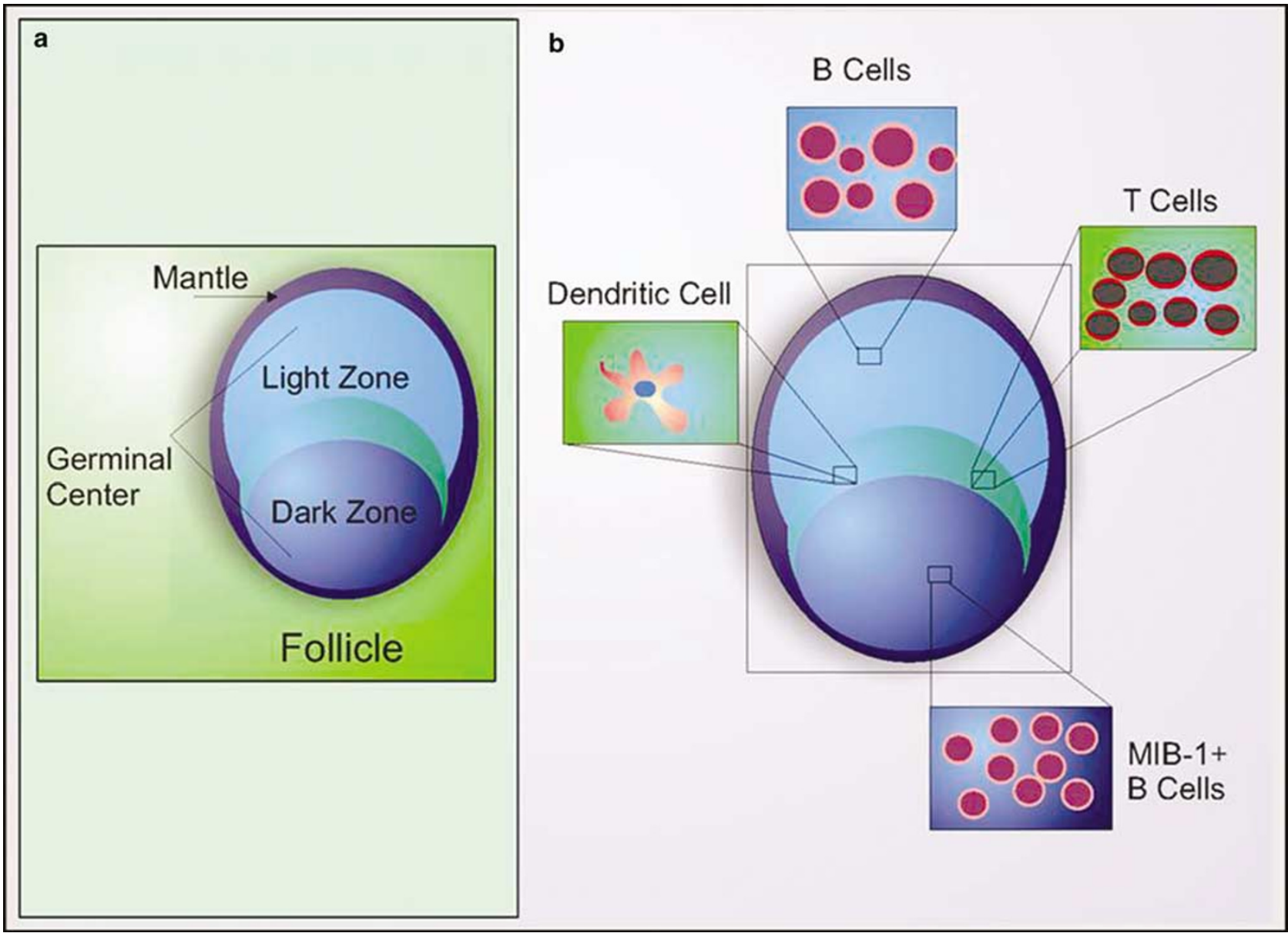

Figure 1 Schematic representation of a secondary lymphoid follicle. (a) Architecture of a reactive lymphoid follicle. (b) Location of cellular subtypes within a reactive follicle.

quantum dots were compared. In our experience, the signal from slides kept at $4^{\circ} \mathrm{C}$ was stable up to 1 year. As controls, either the primary antibody was omitted or an isotype control was used as the primary antibody; both resulted in no observable signal (data not shown).

\section{Confocal Configuration for Higher Order Multiplexing}

Multiplexed immunostaining requires several key components, including well-characterized antibodies, a narrow emission reporter technology, and an imaging system capable of measuring multiple emission spectra reliably. A recently developed technology for confocal microscopy known as emission fingerprinting ${ }^{22}$ was employed to check the emission profile and determine which streptavidin-conjugated quantum dots would be optimal for combined staining of multiple tissue markers. The Zeiss LSM 510 Meta detector was used for this purpose. This detector consists of a 32 channel PMT array that allows the emission spectra to be collected in $10.7 \mathrm{~nm}$ bands across the visible spectrum. With emission fingerprinting, $x-y$ scans were made across a series of wavelengths generating a lambda stack wherein each image in the stack records the emission intensity for each pixel at each wavelength. A linear unmixing algorithm was then applied to the lambda stack using the reference spectra of each of the quantum dots present in a sample. This produces a multifluorescence image with clearly separated channels for each distinct fluorochrome. Double immunostains were performed with the 'test' antibodies (CD20 and MIB-1) previously used in the singles. Figure $2 d$ shows a typical emission fingerprint with good separation of the two emission peaks (565 and $585 \mathrm{~nm}$ ), which corresponded to nuclear (MIB-1, green, Figure 2a) and surface (CD20, red, Figure 2b) immunostains on B cells from a proliferating germinal center (overlay, Figure 2c). In summary, emission fingerprinting enables immunohistochemical two label staining using quantum dot reporters, even if they have emission peaks within $20 \mathrm{~nm}$ of each other.

\section{Quantum Dot Emission Peak Overlap in Higher Order Multiplexing Restricts Quantum Dot Palette}

Emission fingerprint technology clearly distinguished two close quantum dot emitters, 565 and 
MIB-1
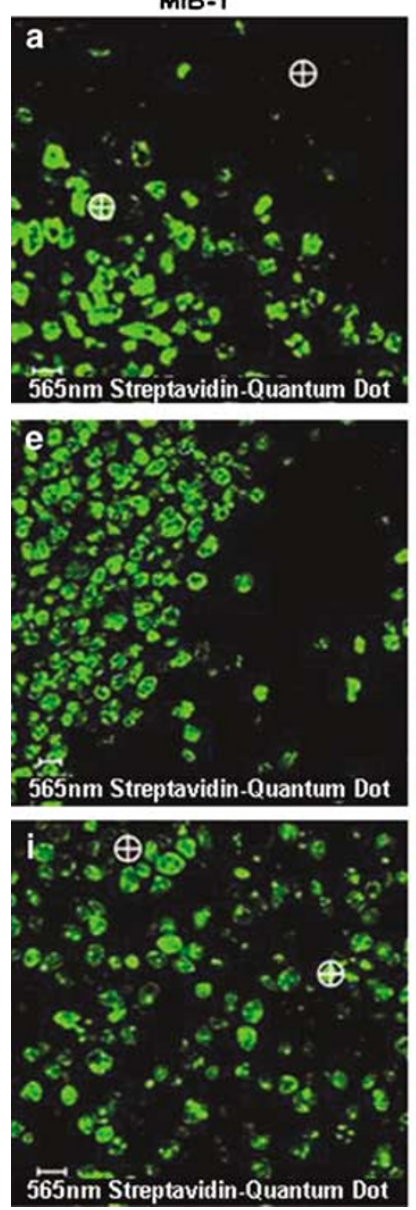

$\operatorname{CD} 20$
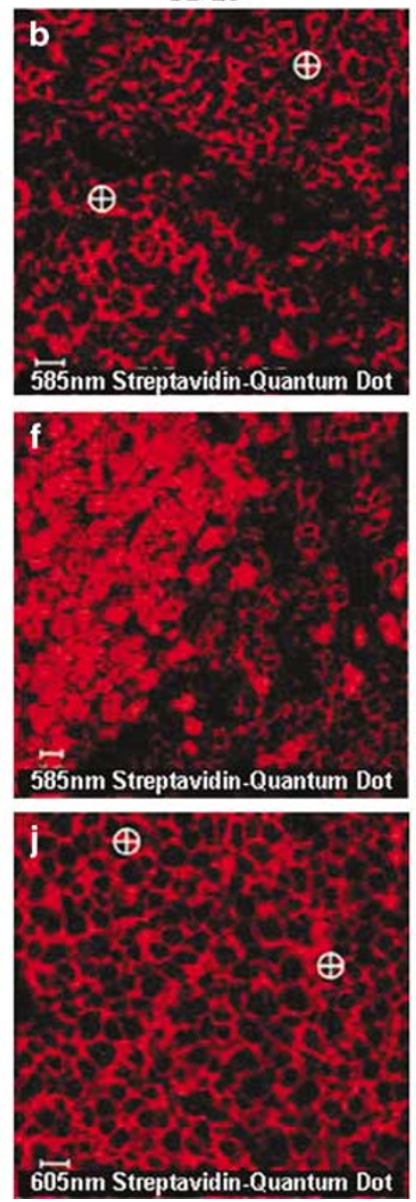

OVERLAY
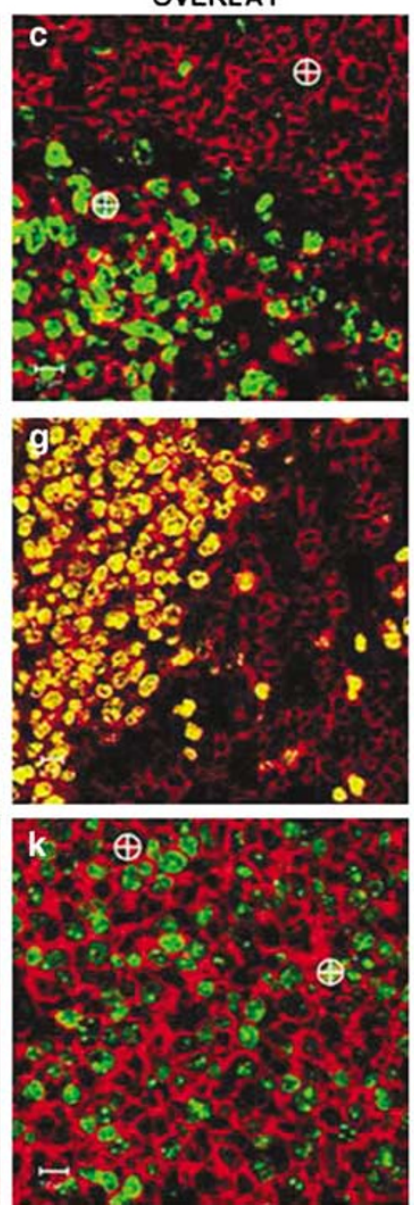
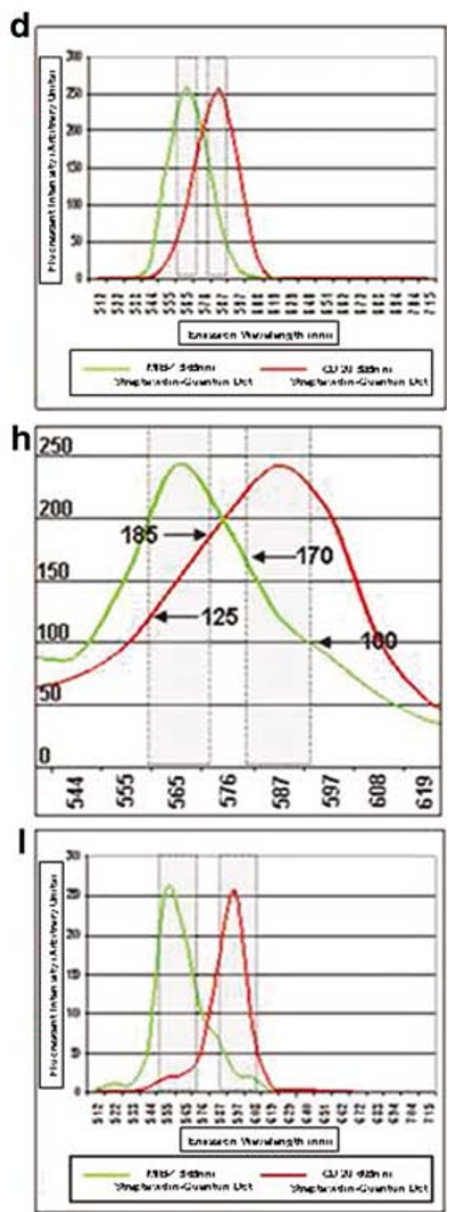

Figure 2 Double stains to optimize band pass filters for confocal configuration. (a-c) Double stained tonsillar tissue section using streptavidin-conjugated quantum dots $565 \mathrm{~nm}$ (green) and $585 \mathrm{~nm}$ (red). Crosshairs denote where the emission curve was generated with Meta detector to perform linear unmixing. (d) Emission fingerprint of streptavidin-conjugated quantum dots $565 \mathrm{~nm}$ (green) and $585 \mathrm{~nm}$ (red). Gray columns represent the band pass filters used for the linear unmixing. (e-g) Same staining as in (a-c), but acquired using the configuration for five streptavidin-conjugated quantum dots plus DAPI. (g) Yellow indicates streptavidin-conjugated quantum dot $565 \mathrm{~nm}$ 'bleeding-through' into the streptavidin-conjugated quantum dot $585 \mathrm{~nm}$ band pass filter. (h) Panel (d) enlarged to show range of pixel intensities that are 'bleeding-through'. (I-k) Double stained tissue section using streptavidin-conjugated quantum dot $565 \mathrm{~nm}$ (green) and $605 \mathrm{~nm}$ (red) and acquired using five streptavidin-conjugated quantum dots plus DAPI configuration. (l) Emission fingerprint of streptavidin-conjugated quantum dots $565 \mathrm{~nm}$ (green) and $605 \mathrm{~nm}$ (red). Gray column represents the band pass filters used. Scale bar is $10 \mu \mathrm{m}$.

$585 \mathrm{~nm}$, when the confocal microscope and detector were configured to detect two signals. However, when the confocal microscope and detector were configured to detect five quantum dot signals plus a DAPI signal, signal overlap between quantum dots within $20 \mathrm{~nm}$ of each other became problematic. More specifically, certain combinations of quantum dots could not be resolved despite the narrower symmetrical emission (Figure 2e-h). The inability to completely separate 565 and $585 \mathrm{~nm}$ (or 585 vs $605 \mathrm{~nm}$, data not shown) was due to the limitations of the Meta detector (10.7 nm bands) and the overlap of the emission profiles (the streptavidin-conjugated quantum dot 565 tailing edge with the leading edge of the streptavidin-conjugated quantum dot 585, Figure 2h). This is morphologically evident, as shown by the aberrant nuclear positivity in Figure $2 \mathrm{f}$ and g. However, when the emission peaks were further apart (more than $20 \mathrm{~nm}$ ), double stains with MIB-1 (565 nm, green) and CD20 (605 nm, red) (Figure 2i and $\mathrm{j}$ ) could be separated using the confocal configuration for five streptavidin-conjugated quantum dots. For this reason, streptavidinconjugated quantum dot $585 \mathrm{~nm}$ was not used in the subsequent experiments when using more than three streptavidin-conjugated quantum dots. The streptavidin-conjugated quantum dot 805 could not be imaged with the Zeiss confocal system, because of its limited wavelength range. However, it could be imaged with the Leica TCS SP2 AOBS system (Leica Microsystems, Exton, PA, USA) due to the longer wavelength range on that system (data not shown). 
Comparison between Directly Labeled Quantum Dots vs Streptavidin-Conjugated Quantum Dots on Formalin-Fixed, Paraffin-Embedded Tissue Sections

As an alternative to streptavidin-conjugated quantum dots, secondary antibodies directly conjugated with quantum dots (525 and $655 \mathrm{~nm}$ ) were tested on consecutive formalin fixed paraffin-embedded tissue sections of reactive tonsil and the fluorescence intensity and signal to background ratio were compared with those obtained with streptavidinconjugated quantum dots. Results for quantum dot $525 \mathrm{~nm}$ are shown in Figure 3 (quantum dot $655 \mathrm{~nm}$, not shown). Due to the reported increased brightness of quantum dots over traditional fluorophores of the same wavelength, ${ }^{4,15}$ we reasoned that directly conjugated quantum dot secondary antibodies would be sufficiently bright to be detected without streptavidin amplification. The aim was to use them as an alternative to streptavidin-conjugated quantum dots or in combination with streptavidinconjugated quantum dots. To test this hypothesis, anti-CD20 antibody was chosen as the primary antibody because of the antigen's high level of surface expression, brightness, and resilience to retrieval conditions. Two different directly conjugated quantum dot secondary antibodies, namely goat anti-mouse IgG (heavy and light chain) quantum dot $525 \mathrm{~nm}$-conjugated and goat anti-mouse IgG (heavy and light chain) quantum dot $655 \mathrm{~nm}$ conjugated were chosen, based on the observed differences of intensity of the quantum dots in previous staining experiments using single streptavidin-conjugated quantum dots (data not shown). They were used on consecutive tissue sections with identical staining conditions. Each pair (ie directly conjugated quantum dot and corresponding streptavidin-conjugated quantum dots) was compared using identical confocal settings and configurations including detector gains, amplifier offsets, amplifier gains, pinholes, objective lens, laser output and power, and main and secondary dichroics. Fluorescence signal intensities and background intensities were quantified using the line profile measurement tool in the Zeiss software where intensity values are graphically presented as fluorescence intensity as arbitrary units vs location of the line $(\mu \mathrm{m})$ in the image. With only one round of amplification (biotin-streptavidin interaction), streptavidin-conjugated quantum dots $525 \mathrm{~nm}$ was approximately three times brighter than directly conjugated quantum dot $525 \mathrm{~nm}$ (Figure 3c). It is of interest to note the differences in background levels between the two directly conjugated quantum dots. Since all other conditions are equal, these differences probably reflect the unique spectral properties of the quantum dots. These experiments were repeated three times with similar results. Because of the weaker signal, the use of directly conjugated quantum dot in multiplex staining was limited. Therefore, only streptavidin-conjugated quantum dots were used in all subsequent experiments.

\section{Multispectral Imaging of Cellular Targets Using Multiple Streptavidin-Conjugated Quantum Dots on a Single Formalin-Fixed, Paraffin-Embedded Tissue}

Determining the optimal combination of quantum dots for immunohistochemical studies was facilitated by using well-studied tissue antigens with known cellular distribution profiles. With higher

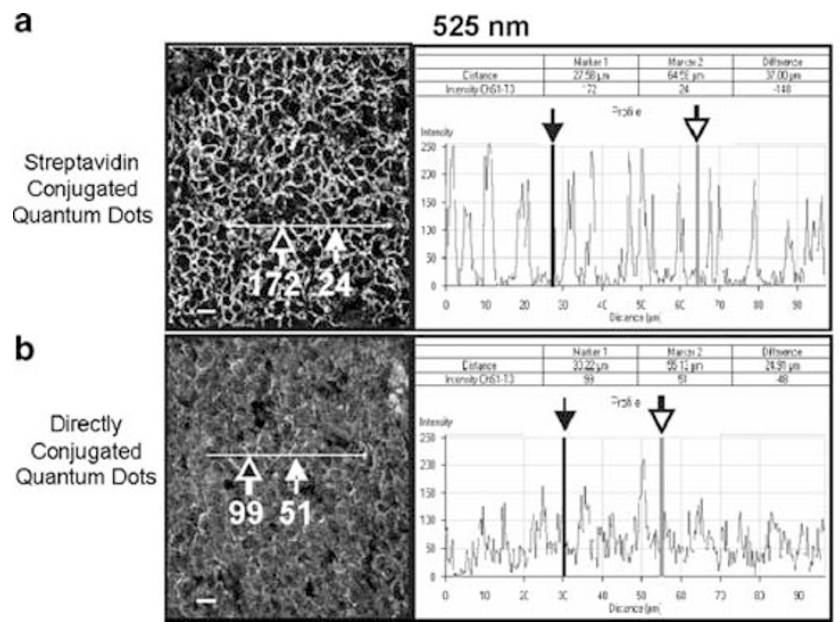

C

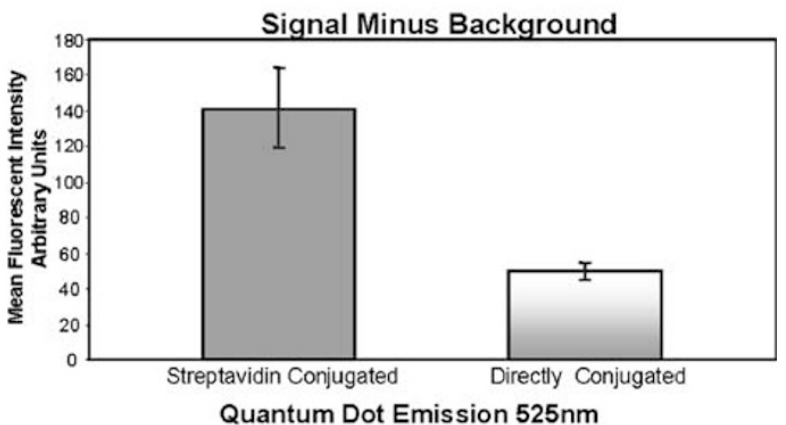

Figure 3 Directly conjugated quantum dot vs streptavidin-conjugated quantum dots. (a, b) Line profile mean of CD 20 signal (black arrows, black line) and mean background (white arrows, white lines). (a) Streptavidin-conjugated quantum dots $525 \mathrm{~nm}$ with signal (black, 172 arbitrary unit) and background (white, 24 arbitrary unit) indicated with arrows. (b) Directly conjugated quantum dot 525 nm with signal (black, 99 arbitrary unit) and background (white, 51 arbitrary unit) indicated with arrows. (c) CD 20 fluorescent intensity mean (minus background) of streptavidin-conjugated quantum dots $525 \mathrm{~nm}$ (dark gray) and directly conjugated quantum dot $525 \mathrm{~nm}$ (light gray). Scale bar is $10 \mu \mathrm{m}$. 
order multiplexing, the order of use of a particular quantum dot must be tailored to account for the affinity of the antibody and the intensity of the quantum dot signal. Essential steps in achieving these multiplexed stains included the addition of an avidin-biotin block step in between the application of the quantum dot and the next sequential primary antibody, as well as extra washes with Tris-buffered saline ( $\mathrm{pH}$ 7.6). However, no improvement in signal over background was found either with addition of $3 \%$ goat serum in all washes, or with added detergent $(0.1 \%$ Tween 20 or NP40), or both. We then increased the number of targets using a combination of pegylated streptavidin-conjugated quantum dots in a sequential manner. Antibodies were chosen not only based on their targets but initially also according to their retrieval conditions. However, this was not always achievable, because some antibodies required a specific retrieval combination. In order to determine whether a specific antibody could be multiplexed with other antibodies, each retrieval method was performed in parallel. In addition, several streptavidin-conjugated quantum dots were tested for each antibody to maximize the intensity of the signal elicited. The aim was to establish the best possible combination of staining conditions, antibodies and streptavidinconjugated quantum dots for analysis using our confocal configuration. With regard to detection, the Meta detector with custom band passes was exclusively used to image all the streptavidin-conjugated quantum dots. Based on earlier experiments, the unique spectral fingerprint for each streptavidinconjugated quantum dot, which provided the framework for designing the custom band passes in the Meta detector, had been identified (for details, see Materials and methods section). Further elements of lymph node cellular architecture were investigated such as $\mathrm{T}$ cells, interdigitating dendritic cells, macrophages, high-endothelial venules, using triple label staining (data not shown) and confirmed the feasibility of our approach.

According to $\mathrm{Wu}$ et $a l,{ }^{15}$ staining of nuclear antigens with quantum dots is often unreliable and inconsistent compared to the staining of surface antigens. Given the positive results with MIB-1, the panel of nuclear targets was expanded. These included Pax-5, BCL-6, and MUM-1 (IRF-4), which are well-characterized B-cell transcription factors that play important roles in B-cell differentiation and germinal center formation. Pax-5 is a member of the highly conserved pair box (PAX)-domain family of transcription factors. It is restricted to cells within the B-cell lineage from pro-B to mature B cells and notably is not expressed in terminally differentiated plasma cells. ${ }^{23,24}$ BCL-6 is a member of the Pox-virus zinc finger/bric-a-brac, tramtrack, broad complex (POZ/BTB) family of transcription factors and is a transcriptional repressor required for germinal center formation. ${ }^{25,26}$ By immunohistochemistry, it is expressed in germinal center B cells as well as in a subset of $\mathrm{T}$ cells associated with the germinal centers. $^{26}$ MUM-1 is a member of the Interferon Regulatory Factors family, known also as IR F-4. This molecule is expressed in terminally differentiated plasma cells and rare positive cells are seen in the light zone in normal reactive germinal centers, where the expression of MUM-1 and BCL-6 is mutually exclusive..$^{27,28}$ The power of this technique for evaluating multiple nuclear targets on formalinfixed, paraffin-embedded tissue sections is shown in Figure 4, where MIB-1, MUM-1 and BCL-6 (Figure $4 \mathrm{a}-\mathrm{c}$ ) were sequentially detected. In reactive germinal centers, the expression pattern between BCL-6 and MUM-1 is mutually exclusive (Figure 4d and e) as previously shown also by Falini, using alkaline phosphatase-anti-alkaline phosphatase (APAAP) ${ }^{28}$ By this triple combination, we also demonstrated that the majority of BCL-6-positive cells are proliferating as shown by coexpression of MIB-1, while the MUM-1-positive cells are not (Figure $4 \mathrm{~d}$ and e).

When performing sequential detection of multiple targets (more than 3), appropriate antibodies and quantum dots were matched and strong-quantum dot:weak-Ab pairs were placed at the beginning of the staining protocol, which minimized loss of signal intensity throughout the lengthy protocol (over $12 \mathrm{~h}$ ). Of note, when using streptavidinconjugated quantum dot $525 \mathrm{~nm}$ and streptavidinconjugated quantum dot $705 \mathrm{~nm}$, it is important to use antibodies of similar sensitivity in order to achieve approximately similar intensities for each pair due to the four track limit for acquisition of our confocal system. Specifically, DAPI and streptavidin-conjugated quantum dot $605 \mathrm{~nm}$ were collected as separate channels in one track, one streptavidinconjugated quantum dot in each of the other two tracks and two streptavidin-conjugated quantum dots (525 and $705 \mathrm{~nm}$ ) in the remaining fourth track. All signals, other than DAPI, were collected with the Meta detector using custom band pass filters. Since the Meta detector does not allow modification of the scanning parameters for each channel collected in the same track, the signal intensities of the two streptavidin-conjugated quantum dots paired in one track should be similar. Again, utilizing a clinically relevant lymphoid tissue, a combination of five streptavidin-conjugated quantum dots plus DAPI is shown in Figure 5 where the reactive germinal center is shown using the following combination of primary antibodies: the B-cell marker CD20 (Figure $5 \mathrm{~b}, 525 \mathrm{~nm}$, red), the B-cell marker IgD (Figure 5c, $565 \mathrm{~nm}$, cyan), the T-cell marker CD3 (Figure 5e, $655 \mathrm{~nm}$, yellow), the macrophage marker CD68 (Figure 5f, $705 \mathrm{~nm}$, white), and MIB-1 (Figure 5d, $605 \mathrm{~nm}$, green). The mantle cells are coexpressing CD20 and IgD as shown by the overlay (Figure 5g, magenta inset). The dark zone is best seen with the proliferation marker MIB-1 as a nuclear stain (Figure $5 \mathrm{~d}$, green) and CD20 (Figure 5b, red) as a surface marker. The emission profile generated from the 

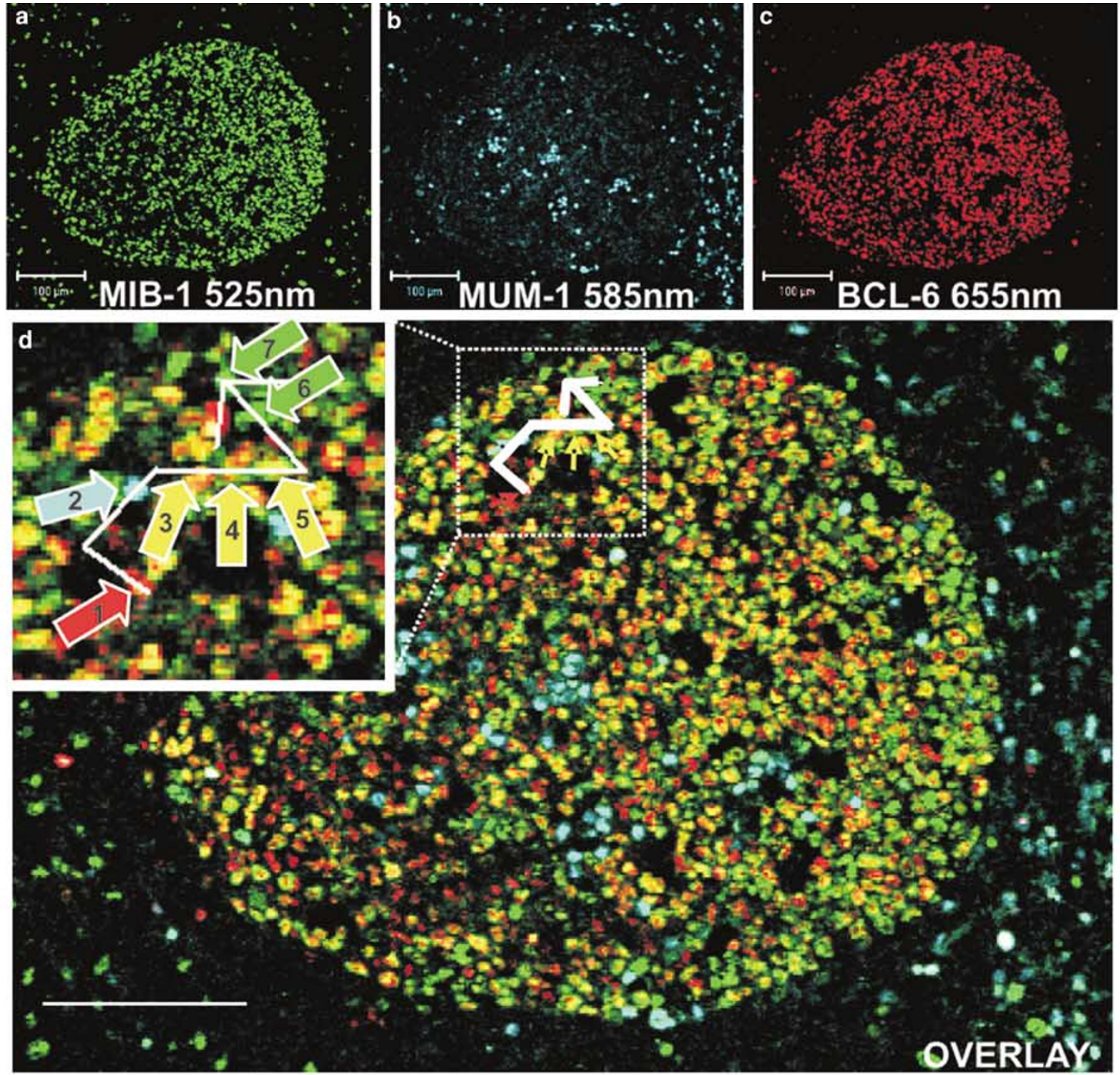

e Intensity Profile

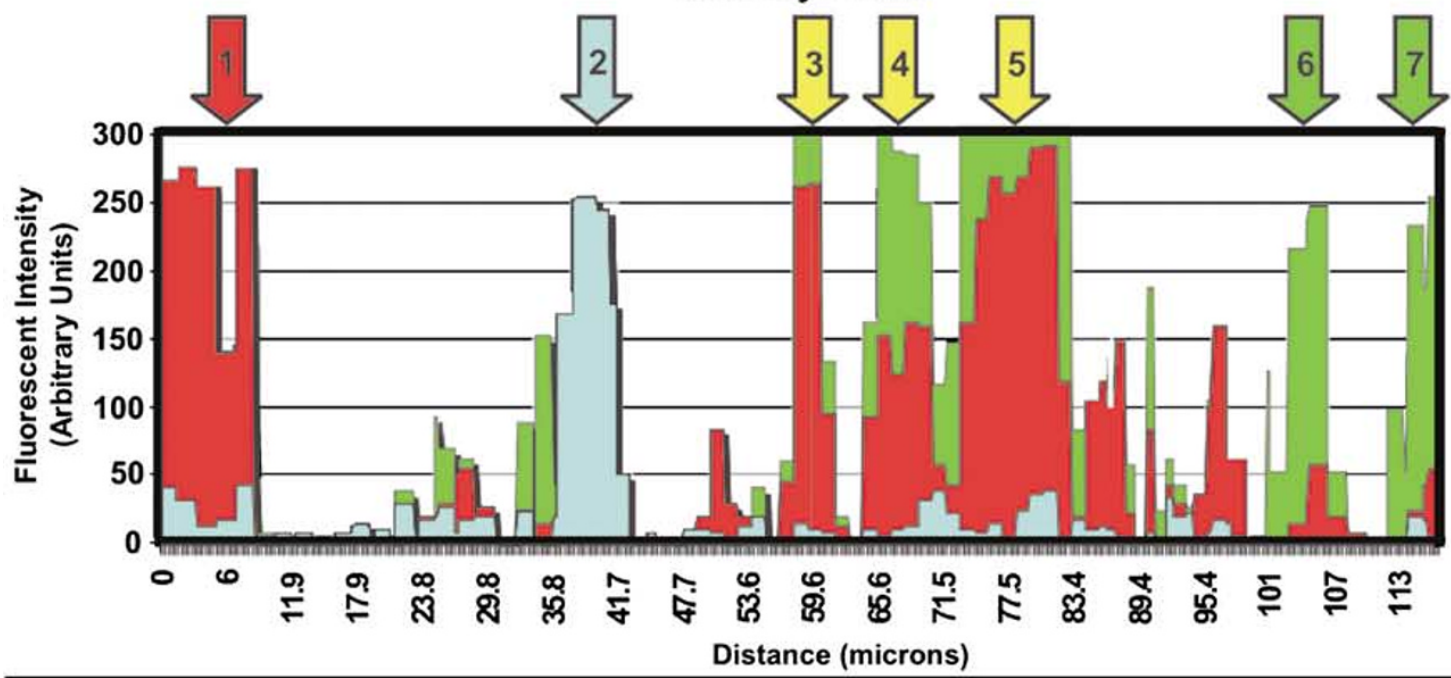

MIB-1 525nm

MUM-1 585nm

BCL-6 655nm

Streptavidin-Quantum Dot 
lambda scan is shown in Figure $5 \mathrm{~h}$. In contrast to the single or double stains using quantum dots, the emission profiles in multiplexed assays are slightly broader.

\section{Discussion}

Disease classification increasingly relies on molecular descriptors, such as the proteins imaged in
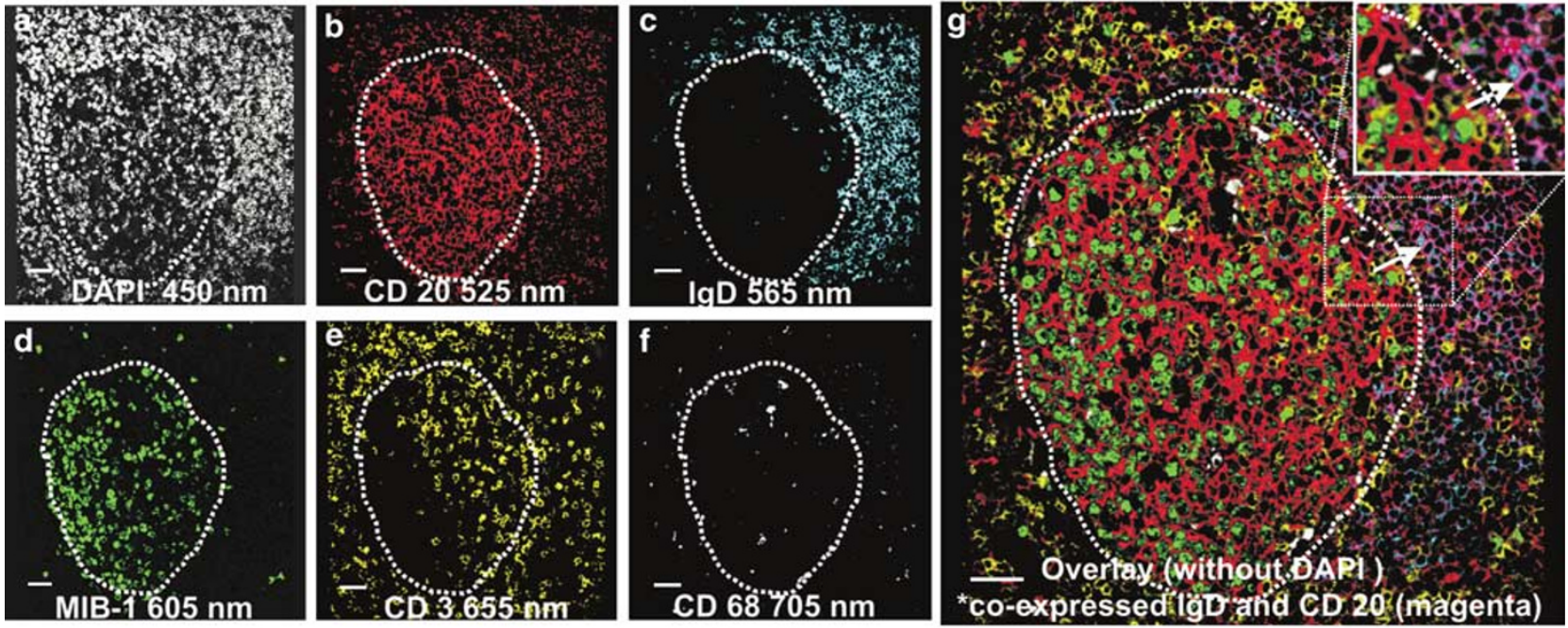

h
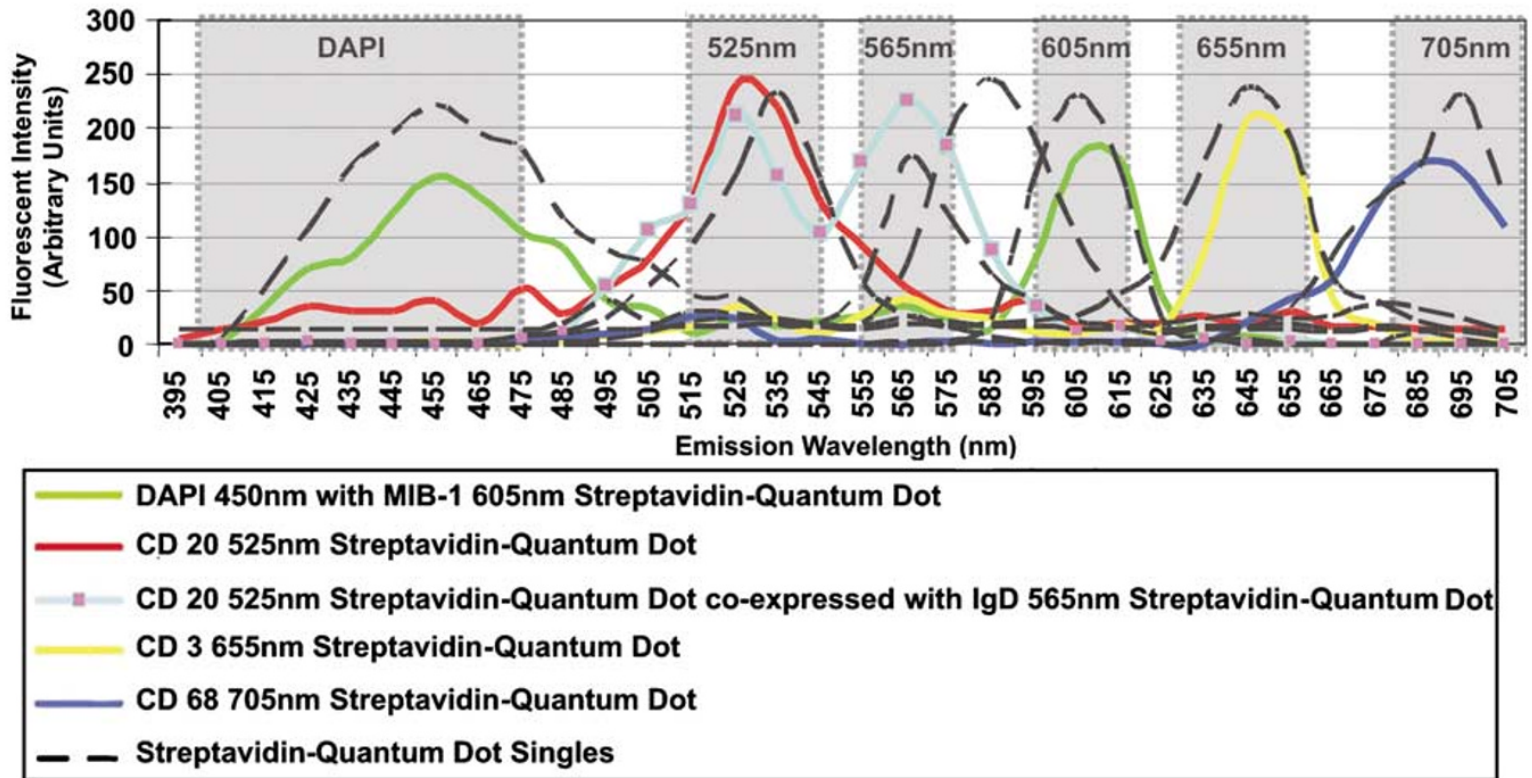

Figure 5 Quintuplet streptavidin-conjugated quantum dots multiplex and emission fingerprint of the multiplexed quantum dots (germinal center outlined with dotted line). (a) DAPI (white) nuclear counter stain (Em. $450 \mathrm{~nm}$ ). (b) CD 20 streptavidin-conjugated quantum dot $525 \mathrm{~nm}$ (red). (c) IgD streptavidin-conjugated quantum dot $565 \mathrm{~nm}$ (cyan). (d) MIB-1 streptavidin-conjugated quantum dot $605 \mathrm{~nm}$ (green). (e) CD 3 streptavidin-conjugated quantum dot $655 \mathrm{~nm}$ (yellow). (f) CD 68 streptavidin-conjugated quantum dot $705 \mathrm{~nm}$ (white). (g) Overlay of (b-f) including IgD (cyan) and CD20 (red) coexpression (magenta, see arrows and inset). (h) Emission profile generated from Lambda scan from 395 to $715 \mathrm{~nm}$. Dotted lines are the single emission profile from DAPI alone or each of the streptavidinconjugated quantum dots alone. Solid colored lines are the indicated emission profiles from the Quintuplet-labeled tonsil section and gray areas define the band pass filters used. Profiles with the same color indicate colocalization. Scale bar is $50 \mu \mathrm{m}$.

Figure 4 Triple stain of germinal center with different nuclear targets. (a) Proliferating B cells in the dark zone, MIB-1 streptavidinconjugated quantum dot $525 \mathrm{~nm}$ (green). (b) Terminally differentiated B cells within germinal center, Mum-1 streptavidin-conjugated quantum dot $585 \mathrm{~nm}$ (cyan). (c) Germinal center B cells positive for Bcl-6 streptavidin-conjugated quantum dot $655 \mathrm{~nm}$ (red). (d) Overlay of all three nuclear targets. White line (enlarged in inset) shows location of line profile measurement. (e) Line profile fluorescence intensity measurement shows coexpression of Bcl-6 and MIB-1 (yellow arrows), Mum-1 expression (cyan arrow), BCL-6 expression (red arrow), and MIB-1 expression (green arrows). Scale bar is $100 \mu \mathrm{m}$. 
this study. Not only is the presence or absence of proteins within cellular populations important but also the level of expression. Proper classification of patients is the cornerstone of proper treatment. As a medical discipline, Pathology has historically relied on innovations in technology to delve more deeply into disease pathophysiology and to develop better diagnostic criteria. Immunohistochemistry is one powerful example of how a basic science tool has been integrated into routine clinical care. As proteomic descriptors of disease processes become better understood, platforms for multiplexed imaging of disease-related proteins will become increasingly important.

Multiplexed immunohistochemical studies currently used for formalin-fixed, paraffin-embedded tissue are limited in their applicability. Chromogenic detection systems rely on the deposition of chromogens, often in close proximity on the tissue, which challenges the detection limits of the human eye. Organic fluorophores provide intense signal, but have wide emission spectra and rapidly photobleach. With a confocal microscope, staining of formalin-fixed, paraffin-embedded tissue for more than four proteins with conventional fluorophores is more problematic due to wider emission peaks, the need for multiple laser lines for excitation, and existing band pass filters and dichroics on the system. ${ }^{29}$ In addition, most band pass filters are too broad to separate such closely emitting fluorophores. These problems are magnified further on a conventional fluorescence microscope where laser lines are replaced by a mercury lamp and where both excitation and emission are controlled by band pass filters. The advantages of using quantum dots on tissue sections over traditional immunofluorescence are evident when multiplexing over three colors, when localization of rare targets requires extended time at the fluorescent microscope, and when repeated scans over time are necessary.

In this study, Quantum $\operatorname{dot}^{\circledR}$ technology was found to overcome many of the obstacles encountered with conventional reporter technologies when viewing multiple tissue targets at the same time. We successfully stained formalin-fixed, paraffin-embedded tissue tonsil sections with up to five different streptavidin-conjugated quantum dots simultaneously. This multispectral staining exhibited high specificity, low background and high emission intensity. Moreover, the quantum dot signal is quite stable, resisting photobleaching. ${ }^{8}$ Within the context of a clinical tool, the ability to repeatedly image a tissue is often required. This study demonstrates a significant step in vetting quantum dots as probes in this system for clinically relevant proteomic targets. Numerous factors, including the imaging microscope, antigen retrieval, order of antibody staining, quantum dot intensity, and the sensitivity of the antibody must be thoroughly evaluated in order to configure a staining method that yields the best results. As antibodies directly conjugated to quantum dots become available, a more streamlined multiplexed assay will be possible. Further, hyperspectral imaging ${ }^{17}$ of quantum dots has been performed previously. This tool will likely increase the ability to resolve numerous spectral emission patterns simultaneously, which will increase the number of quantum dots that can be used per slide. In the future, for particular disease conditions, a panel of antibodies labeled with quantum dot tags could potentially be used to further stratify patients as a component of the modern molecular pathology laboratory.

\section{Acknowledgement}

This work was funded by the NIH/NCI intramural program.

\section{References}

1 Michalet X, Pinaud FF, Bentolila LA, et al. Quantum dots for live cells, in vivo imaging, and diagnostics. Science 2005;307:538-544.

2 Akerman ME, Chan WC, Laakkonen P, et al. Nanocrystal targeting in vivo. Proc Natl Acad Sci USA 2002; 99:12617-12621.

3 Gao X, Yang L, Petros JA, et al. In vivo molecular and cellular imaging with quantum dots. Curr Opin Biotechnol 2005;16:63-72.

4 Chan WC, Nie S. Quantum dot bioconjugates for ultrasensitive nonisotopic detection. Science 1998; 281:2016-2018.

5 Alivisatos P. The use of nanocrystals in biological detection. Nat Biotechnol 2004;22:47-52.

6 Jaiswal JK, Simon SM. Potentials and pitfalls of fluorescent quantum dots for biological imaging. Trends Cell Biol 2004;14:497-504.

7 Medintz IL, Uyeda HT, Goldman ER, et al. Quantum dot bioconjugates for imaging, labelling and sensing. Nat Mater 2005;4:435-446.

8 Alivisatos A, Gu W, Larabell C. Quantum dots as cellular probes. Annu Rev Biomed Eng 2005;7:55-76.

9 Gao X, Nie S. Molecular profiling of single cells and tissue specimens with quantum dots. Trends Biotechnol 2003;21:371-373.

10 Goldman ER, Clapp AR, Anderson GP, et al. Multiplexed toxin analysis using four colors of quantum dot fluororeagents. Anal Chem 2004;76:684-688.

11 Lidke DS, Nagy P, Heintzmann R, et al. Quantum dot ligands provide new insights into erbB/HER receptormediated signal transduction. Nat Biotechnol 2004;22: 198-203.

12 Voura EB, Jaiswal JK, Mattoussi H, et al. Tracking metastatic tumor cell extravasation with quantum dot nanocrystals and fluorescence emission-scanning microscopy. Nat Med 2004;10:993-998.

13 Nisman R, Dellaire G, Ren Y, et al. Application of quantum dots as probes for correlative fluorescence, conventional, and energy-filtered transmission electron microscopy. J Histochem Cytochem 2004;52: 13-18. 
14 Giepmans BNG, Deerinck TJ, Smarr BL, et al. Correlated light and electron microscopic imaging of multiple endogenous proteins using quantum dots. Nat Methods 2005;2:743-749.

$15 \mathrm{Wu}$ X, Liu H, Liu J, et al. Immunofluorescent labeling of cancer marker Her2 and other cellular targets with semiconductor quantum dots. Nat Biotechnol 2003;21: 41-46.

16 Dubertret B, Skourides P, Norris DJ, et al. In vivo imaging of quantum dots encapsulated in phospholipid micelles. Science 2002;298:1759-1762.

17 Geho D, Lahar N, Gurnani P, et al. Pegylated, steptavidin-conjugated quantum dots are effective detection elements for reverse-phase protein microarrays. Bioconjug Chem 2005;16:559-566.

18 Ness JM, Akhtar RS, Latham CB, et al. Combined tyramide signal amplification and quantum dots for sensitive and photostable immunofluorescence detection. J Histochem Cytochem 2003;51:981-987.

19 MacLennan I. Germinal centers. Annu Rev Immunol 1994;12:117-139.

20 Gerdes J, Schwab U, Lemke H, et al. Production of a mouse monoclonal antibody reactive with a human nuclear antigen associated with cell proliferation. Int J Cancer 1983;31:13-20.

21 Cattoretti G, Becker MH, Key G, et al. Monoclonal antibodies against recombinant parts of the Ki-67 antigen (MIB 1 and MIB 3) detect proliferating cells in microwave-processed formalin-fixed paraffin sections. J Pathol 1992;168:357-363.
22 Dickinson ME, Bearman G, Tille S, et al. Multi-spectral imaging and linear unmixing add a whole new dimension to laser scanning fluorescence microscopy. Biotechniques 2001;31:1272, 1274-1276, 1278.

23 Barberis A, Widenhorn K, Vitelli L, et al. A novel Bcell lineage-specific transcription factor present at early but not late stages of differentiation. Genes Dev 1990;4:849-859.

24 Lin K-I, Angelin-Duclos C, Kuo T, et al. Blimp-1dependent repression of Pax-5 is required for differentiation of $\mathrm{B}$ cells to immunoglobulin M-secreting plasma cells. Mol Cell Biol 2002;22:4771-4780.

25 Chang C-C, Ye B-H, Chaganti R, et al. BCL-6, a POZ/ zinc-finger protein, is a sequence specific transcriptional repressor. Proc Natl Acad Sci USA 1996;93: 6947-6952.

26 Cattoretti G, Chang C-C, Checova K, et al. BCL-6 protein is expressed in germinal-center B cells. Blood 1995;86:45-53.

27 Angelin-Duclos C, Cattoretti G, Lin K-L, et al. Commitment of $B$ lymphocytes to a plasma cell fate is associated with Blimp-1 expression in vivo. J Immunol 2000;165:5462-5471.

28 Falini B, Fizzotti M, Pucciarini A, et al. A monoclonal antibody (MUM1p) detects expression of the MUM1/ IRF4 protein in a subset of germinal center B cells, plasma cells, and activated T cells. Blood 2000;95: 2084-2092.

29 Lichtman J, Conchello J-A. Fluorescence microscopy. Nat Methods 2005;2:910-919. 